\title{
An Efficient and Green Method for Synthesis of 2,4,5-Triarylimidazoles without Use of Any Solvent, Catalyst, or Solid Surface
}

\author{
Swati Samanta, Saheli Sarkar, Rammohan Pal, and Asok K. Mallik \\ Department of Chemistry, Jadavpur University, Kolkata 700 032, India \\ Correspondence should be addressed to Asok K. Mallik; mallikak52@yahoo.co.in
}

Received 31 August 2013; Accepted 31 October 2013

Academic Editor: Jason Belitsky

Copyright (c) 2013 Swati Samanta et al. This is an open access article distributed under the Creative Commons Attribution License, which permits unrestricted use, distribution, and reproduction in any medium, provided the original work is properly cited.

An efficient and green method for synthesis of 2,4,5-triarylimidazoles without use of any catalyst or solvent has been developed simply by heating $\left(\right.$ at $\left.130^{\circ} \mathrm{C}\right)$ of mixtures of 1,2-diketone, aromatic aldehyde, and ammonium acetate in 1:1:3 mole ratio.

\section{Introduction}

Multicomponent reactions (MCRs) have emerged as a powerful tool for convergent synthesis of many complex organic molecules [1-6]. They are one-pot processes bringing together three or more components in a particular sequence of reactions and show high atom economy and remarkable selectivity. Because of their operational simplicity, MCRs have occupied a very prominent place in diversity oriented synthesis which is an important requirement for drug discovery. The imidazole nucleus is a rich source for getting biologically important organic molecules. Compounds containing imidazole moiety show a range of pharmacological properties and play important roles in biochemical processes. Various substituted imidazoles act as inhibitors of P38 MAP kinase [7] and B-Raf kinase [8], glucagon receptors [9], pesticides [10], fungicides [10], herbicides [11], and antitumor [12], antiinflammatory [13], and antithrombotic [14] agents. Moreover, they are used in photography as photosensitive compounds [15]. 2,4,5-Triarylimidazoles (3) form an important group of substituted imidazoles having many of the above biological activities and material properties. Retrosynthetic analysis of 3 suggests the readily available compounds aromatic 1,2-diketones, aromatic aldehydes, and ammonia as their precursors. This has led to the development of a large number of synthetic methods for $\mathbf{3}$ using these simple starting materials. Almost all of these methods use ammonium acetate as the ammonia source. Many of the reported methods require long reaction time and use of expensive catalysts and organic solvents [16-23]. The current literature shows that there has been a growing trend towards green synthesis of these compounds [24, 25]. However, in such reported green methods, also use of catalysts or organic solvents could not be avoided. The current trend towards development of catalystfree and solvent-free reaction conditions for organic synthesis $[26,27]$ encouraged us to study the same reaction under thermal condition without using any solvent or catalyst. The remarkable success in this endeavor is presented herein.

\section{Results and Discussion}

Our present method involves subjecting of an intimate mixture of 1,2-diketone, aromatic aldehyde, and ammonium acetate in $1: 1: 3$ mole ratio directly to heat $\left(130^{\circ} \mathrm{C}, 3-6 \mathrm{~h}\right)$. A range of structurally diverse aldehydes belonging to the categories aromatic and heterocyclic aldehydes were taken (Scheme 1). To our delight, the target compounds were obtained in good to very good yield in this method for all the combinations. The yields of the products are presented in Table 1.

In the method being reported, it was a common observation that the reactions were very clean and no side product was formed in any run. In fact, the crude products obtained were of high purity and did not require any chromatographic separation. Their crystallization from ethanol provided analytically pure samples. More significantly, the whole operation 


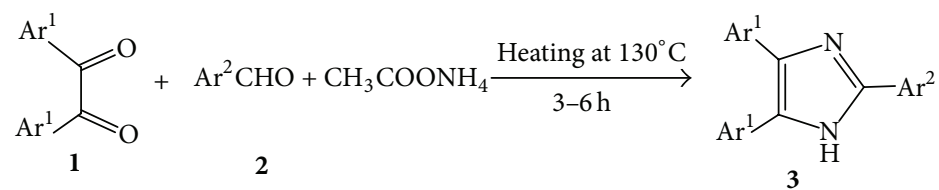

Scheme 1: Synthesis of 2,4,5-triarylimidazoles (3) under catalyst-free and solvent-free condition. $\mathrm{Ar}^{1}=\mathrm{C}_{6} \mathrm{H}_{5} ; 4-\mathrm{CH}_{3}-\mathrm{C}_{6} \mathrm{H}_{4} ; \mathrm{Ar}^{2}=\mathrm{C}_{6} \mathrm{H}_{5}$; $4-\mathrm{CH}_{3}-\mathrm{C}_{6} \mathrm{H}_{4} ; 4-\mathrm{Cl}-\mathrm{C}_{6} \mathrm{H}_{4} ; 4-\mathrm{Br}-\mathrm{C}_{6} \mathrm{H}_{4} ; 4-\mathrm{CH}_{3} \mathrm{O}-\mathrm{C}_{6} \mathrm{H}_{4} ; 3,4-\left(\mathrm{OCH}_{2} \mathrm{O}\right) \mathrm{C}_{6} \mathrm{H}_{3} ; 3-\mathrm{O}_{2} \mathrm{~N}-\mathrm{C}_{6} \mathrm{H}_{4} ; 4-\left(\mathrm{CH}_{3}\right)_{2} \mathrm{~N}-\mathrm{C}_{6} \mathrm{H}_{4} ; 3-\mathrm{CH}_{3} \mathrm{O}, 4-\mathrm{HO}-\mathrm{C}_{6} \mathrm{H}_{3} ; 2-$ Thienyl; 3-Pyridyl.

did not require any solvent, organic or inorganic, at any stage. Furthermore, the reaction condition has been found to be mild enough to tolerate a variety of functionalities such as $\mathrm{NO}_{2}, \mathrm{Cl}, \mathrm{OH}$, and $\mathrm{OMe}$.

\section{Conclusion}

We report here a very simple and efficient green method for synthesis of 2,4,5-triarylimidazoles avoiding the use of any solvent, catalyst, and solid surface.

\section{Experimental}

4.1. General. Melting points were recorded on a Köfler block. IR spectra were recorded on a Perkin Elmer FTIR spectrophotometer (Spectrum BX II) in $\mathrm{KBr}$ pellets. ${ }^{1} \mathrm{H}$ $\mathrm{NMR}$ spectra and ${ }^{13} \mathrm{C}$ NMR spectra were recorded in $\mathrm{CDCl}_{3}$ on a Bruker AV-300 (300 MHz) spectrometer. Analytical samples were routinely dried in vacuo at room temperature. Microanalytical data were recorded on two Perkin-Elmer 2400 Series II C, H, N analyzers. Mass spectra were measured in the following ways FAB-MS [Jeol the M Station JMS.700]. TLC was performed with silica gel G made by SRL Pvt. Ltd. Petroleum ether had the boiling range $60-80^{\circ} \mathrm{C}$.

4.2. General Method for Synthesis of 2,4,5-Triarylimidazoles (3). In a typical experiment, an intimate mixture of benzil ( $1 \mathrm{mmol})$, aromatic aldehyde $(1 \mathrm{mmol})$, and ammonium acetate $(3 \mathrm{mmol})$ was taken in a round-bottom flask $(50 \mathrm{~mL})$ fitted with a $\mathrm{CaCl}_{2}$-guard tube and the flask was heated in an oil bath at $130^{\circ} \mathrm{C}$. Initially, the reaction mixture melted and after some time (ca. 1-3 h) it began to solidify. When the reaction mixture solidified totally (time period mentioned in Table 1), it was cooled to room temperature and to it water $(20 \mathrm{~mL})$ was added. The resulting solid mass was crushed and it was filtered, and the residue was washed with water and then dried. The crude product so obtained was crystallized from ethanol.

All of the products 3a-n were known compounds and were identified by comparison of their physical and spectral data with those reported in the literature. The spectral data recorded by us are given below.

4.2.1. Compound 3a. Colorless needles, ${ }^{1} \mathrm{H}$ NMR $(300 \mathrm{MHz}$, $\mathrm{CDCl}_{3}, \delta / \mathrm{ppm}$ ): and 7.95 (br. d, $2 \mathrm{H}, J=8.1 \mathrm{~Hz}$ ), 7.59 (br. d, $4 \mathrm{H}, J=7.8 \mathrm{~Hz}$ ), $7.28-7.48(\mathrm{~m}, 9 \mathrm{H})$; Anal. Calcd. for $\mathrm{C}_{21} \mathrm{H}_{16} \mathrm{~N}_{2}$
(296.37): C, 85.11; H, 5.44; N, 9.45. Found: C, 85.26; H, 5.72; N, 9.31 .

4.2.2. Compound 3b. Colorless needles, IR $\left(\mathrm{KBr}, \mathrm{cm}^{-1}\right)$ : 3414 , 3027, 2363, 1654, 1601, 1493, 1449, 1381; ${ }^{1} \mathrm{H}$ NMR $(300 \mathrm{MHz}$, $\left.\mathrm{CDCl}_{3}, \delta / \mathrm{ppm}\right): 7.86(\mathrm{~d}, 2 \mathrm{H}, J=8.1 \mathrm{~Hz}), 7.50$ (br. d, $4 \mathrm{H}$, $J \approx 7.8 \mathrm{~Hz}), 7.21-7.31(\mathrm{~m}, 8 \mathrm{H}), 2.38\left(\mathrm{~s}, 3 \mathrm{H}, \mathrm{CH}_{3}\right) ;{ }^{13} \mathrm{C} \mathrm{NMR}$ $\left(75 \mathrm{MHz}, \mathrm{CDCl}_{3}, \delta / \mathrm{ppm}\right): 146.2,138.9,132.9,129.6,128.6$, 127.8, 127.4, 127.1, 125.2, 21.3; FAB MS $(\mathrm{M}+\mathrm{H})^{+}$: Calcd. 311.3. Found 311.3; Anal. Calcd. for $\mathrm{C}_{22} \mathrm{H}_{18} \mathrm{~N}_{2}$ (310.39): C, 85.13; $\mathrm{H}$, 5.85; N, 9.03. Found: C, 84.86; H, 5.75; N, 8.82.

4.2.3. Compound 3c. Colorless needles, ${ }^{1} \mathrm{H}$ NMR $(300 \mathrm{MHz}$, $\mathrm{CDCl}_{3}, \delta / \mathrm{ppm}$ ): 7.83 (d, $2 \mathrm{H}, J=8.7 \mathrm{~Hz}$ ), 7.53 (br. d, $4 \mathrm{H}, J \approx$ $7.0 \mathrm{~Hz}), 7.28-7.40(\mathrm{~m}, 8 \mathrm{H})$.

4.2.4. Compound 3d. Colorless needles, ${ }^{1} \mathrm{H}$ NMR $(300 \mathrm{MHz}$, $\left.\mathrm{CDCl}_{3}, \delta / \mathrm{ppm}\right): 7.78(\mathrm{~d}, 2 \mathrm{H}, J=8.5 \mathrm{~Hz}), 7.54-7.58(\mathrm{~m}, 6 \mathrm{H})$, $7.28-7.38(\mathrm{~m}, 6 \mathrm{H})$.

4.2.5. Compound 3e. Colorless needles, ${ }^{1} \mathrm{H}$ NMR $(300 \mathrm{MHz}$, $\mathrm{CDCl}_{3}, \delta / \mathrm{ppm}$ ): 7.88 (br. d, $2 \mathrm{H}, J=8.4 \mathrm{~Hz}$ ), 7.49 (br. d, $4 \mathrm{H}$, $J \approx 6.6 \mathrm{~Hz}), 7.24-7.30(\mathrm{~m}, 6 \mathrm{H}), 6.93(\mathrm{~d}, 2 \mathrm{H}, J=9 \mathrm{~Hz}), 3.82(\mathrm{~s}$, $3 \mathrm{H})$.

4.2.6. Compound 3f. Colorless needles, ${ }^{1} \mathrm{H}$ NMR $(300 \mathrm{MHz}$, $\mathrm{d}_{6}$-DMSO, $\left.\delta / \mathrm{ppm}\right): 12.49$ (s, 1H, NH), 7.21-7.63 (m, 12H), 7.03 $(\mathrm{d}, 1 \mathrm{H}, J=8.4), 6.08\left(\mathrm{~s}, 2 \mathrm{H},-\mathrm{OCH}_{2} \mathrm{O}-\right) ;{ }^{1} \mathrm{H}$ NMR $(300 \mathrm{MHz}$, $\mathrm{CDCl}_{3}, \delta / \mathrm{ppm}$ ): 7.52 (br. d, $4 \mathrm{H}, J \approx 7.2 \mathrm{~Hz}$ ), 7.39 (d, $1 \mathrm{H}, J=$ $8.2 \mathrm{~Hz}$ ), 7.46 (br. s, $1 \mathrm{H}), 7.26-7.33(\mathrm{~m}, 5 \mathrm{H}), 6.83$ (d, $1 \mathrm{H}, J=$ $8.0 \mathrm{~Hz}), 6.00\left(\mathrm{~s}, 2 \mathrm{H},-\mathrm{OCH}_{2} \mathrm{O}-\right)$.

4.2.7. Compound 3g. Yellow needles, ${ }^{1} \mathrm{H}$ NMR $\left(300 \mathrm{MHz}, \mathrm{d}_{6}{ }^{-}\right.$ DMSO, $\delta / \mathrm{ppm}): 13.08$ (s, 1H), $8.94(\mathrm{~s}, 1 \mathrm{H}), 8.50$ (d, $1 \mathrm{H}, J=$ $7.8 \mathrm{~Hz}), 8.19(\mathrm{~d}, 1 \mathrm{H}, J=8.1 \mathrm{~Hz}), 7.76(\mathrm{t}, 1 \mathrm{H}, J=7.8 \mathrm{~Hz}), 7.32-$ $7.53(\mathrm{~m}, 10 \mathrm{H})$.

4.2.8. Compound 3h. Colorless needles, ${ }^{1} \mathrm{H}$ NMR $(300 \mathrm{MHz}$, $\left.\mathrm{CDCl}_{3}, \delta / \mathrm{ppm}\right): 7.82(\mathrm{~d}, 2 \mathrm{H}, J=7.7 \mathrm{~Hz}), 7.52-7.54(\mathrm{~m}, 4 \mathrm{H})$, $7.26-7.33(\mathrm{~m}, 6 \mathrm{H}), 6.74(\mathrm{~d}, 2 \mathrm{H}, J=7.8 \mathrm{~Hz}), 2.99\left(\mathrm{~s}, 6 \mathrm{H}, \mathrm{NMe}_{2}\right)$.

4.2.9. Compound 3i. Colorless needles, ${ }^{1} \mathrm{H}$ NMR $(300 \mathrm{MHz}$, $\mathrm{CDCl}_{3}, \delta / \mathrm{ppm}$ ): 12.40 (br. s, $1 \mathrm{H}, \mathrm{NH}$ ), 9.24 (br. s, $1 \mathrm{H}, \mathrm{OH}$ ), 7.19-7.54 (m, 11H), 7.61 (br. s, $1 \mathrm{H}), 6.84(\mathrm{~d}, 1 \mathrm{H}, J=8.2 \mathrm{~Hz}), 3.84$ $\left(\mathrm{s}, 3 \mathrm{H}, \mathrm{OCH}_{3}\right)$. 
TABLE 1: Synthesis of 2,4,5-triarylimidazoles (3) under catalyst-free and solvent-free conditions.

\begin{tabular}{|c|c|c|c|c|c|c|}
\hline \multirow{2}{*}{ Entry } & \multicolumn{2}{|c|}{ Starting materials } & \multirow{2}{*}{ Product } & \multirow{2}{*}{ Time (h) } & \multirow{2}{*}{ Yield (\%) } & \multirow{2}{*}{$\begin{array}{l}\text { M. P. (Lit.) } \\
\text { reference }\end{array}$} \\
\hline & 1 with $\mathrm{Ar}^{1}=$ & 2 with $\mathrm{Ar}^{2}=$ & & & & \\
\hline 1 & $\mathrm{C}_{6} \mathrm{H}_{5}$ & $\mathrm{C}_{6} \mathrm{H}_{5}$ & $3 a$ & 3 & 80 & $\begin{array}{c}272-273(272-274) \\
{[19]}\end{array}$ \\
\hline 2 & $\mathrm{C}_{6} \mathrm{H}_{5}$ & $4-\mathrm{CH}_{3}-\mathrm{C}_{6} \mathrm{H}_{4}$ & $3 b$ & 3 & 75 & $\begin{array}{c}227-228(227-228) \\
{[19]}\end{array}$ \\
\hline 3 & $\mathrm{C}_{6} \mathrm{H}_{5}$ & $4-\mathrm{Cl}-\mathrm{C}_{6} \mathrm{H}_{4}$ & $3 c$ & 3 & 78 & $\begin{array}{c}261-262(261-262) \\
{[19]}\end{array}$ \\
\hline 4 & $\mathrm{C}_{6} \mathrm{H}_{5}$ & $4-\mathrm{Br}-\mathrm{C}_{6} \mathrm{H}_{4}$ & $3 d$ & 3 & 81 & $\begin{array}{c}245-246(244-246) \\
{[19]}\end{array}$ \\
\hline 5 & $\mathrm{C}_{6} \mathrm{H}_{5}$ & $4-\mathrm{CH}_{3} \mathrm{O}-\mathrm{C}_{6} \mathrm{H}_{4}$ & $3 e$ & 3 & 76 & $\begin{array}{c}226-227(227-228) \\
{[19]}\end{array}$ \\
\hline 6 & $\mathrm{C}_{6} \mathrm{H}_{5}$ & $3,4-\left(\mathrm{OCH}_{2} \mathrm{O}\right) \mathrm{C}_{6} \mathrm{H}_{3}$ & $3 f$ & 3 & 73 & $\begin{array}{c}248-250(248-250) \\
{[19]}\end{array}$ \\
\hline 7 & $\mathrm{C}_{6} \mathrm{H}_{5}$ & $3-\mathrm{O}_{2} \mathrm{~N}-\mathrm{C}_{6} \mathrm{H}_{4}$ & $3 g$ & 3 & 90 & $>300(>290)[19]$ \\
\hline 8 & $\mathrm{C}_{6} \mathrm{H}_{5}$ & 4- $\left(\mathrm{CH}_{3}\right)_{2} \mathrm{~N}-\mathrm{C}_{6} \mathrm{H}_{4}^{-}$ & $3 h$ & 3 & 45 & $\begin{array}{c}256-258(257-258) \\
{[19]}\end{array}$ \\
\hline 9 & $\mathrm{C}_{6} \mathrm{H}_{5}$ & $3-\mathrm{CH}_{3} \mathrm{O}, 4-\mathrm{HO}-\mathrm{C}_{6} \mathrm{H}_{3}-$ & $3 \mathbf{i}$ & 5 & 67 & $165-167$ (166) [28] \\
\hline 10 & $\mathrm{C}_{6} \mathrm{H}_{5}$ & 2-Thienyl & $3 \mathbf{j}$ & 4 & 67 & $\begin{array}{c}261-263(260-262) \\
{[29]}\end{array}$ \\
\hline 11 & $\mathrm{C}_{6} \mathrm{H}_{5}$ & 3-Pyridyl & $3 k$ & 3 & 66 & $\begin{array}{c}245-247(244-246) \\
{[30]}\end{array}$ \\
\hline 12 & $4-\mathrm{CH}_{3}-\mathrm{C}_{6} \mathrm{H}_{4}^{-}$ & $\mathrm{C}_{6} \mathrm{H}_{5}$ & 31 & 5 & 59 & $252-253(254)[31]$ \\
\hline 13 & $4-\mathrm{CH}_{3}-\mathrm{C}_{6} \mathrm{H}_{4}-$ & 4-Cl- $\mathrm{C}_{6} \mathrm{H}_{4}$ & $3 m$ & 5 & 64 & $\begin{array}{c}262-263(263-264) \\
{[32]}\end{array}$ \\
\hline 14 & $4-\mathrm{CH}_{3}-\mathrm{C}_{6} \mathrm{H}_{4}-$ & $4-\mathrm{CH}_{3} \mathrm{O}-\mathrm{C}_{6} \mathrm{H}_{4}$ & $3 n$ & 6 & 61 & $\begin{array}{c}250-251(250-251) \\
{[33]}\end{array}$ \\
\hline
\end{tabular}

4.2.10. Compound 3j. Colorless needles, ${ }^{1} \mathrm{H}$ NMR $(300 \mathrm{MHz}$, $\mathrm{CDCl}_{3}, \delta / \mathrm{ppm}$ ): 7.70 (br. d, $1 \mathrm{H}, J \approx 3 \mathrm{~Hz}$ ), 7.43-7.46 (m, $4 \mathrm{H}$ ), $7.31(\mathrm{dd}, 1 \mathrm{H}, J=5.1$ and $1.0 \mathrm{~Hz}), 7.24-7.28(\mathrm{~m}, 6 \mathrm{H}), 7.04(\mathrm{dd}$, $1 \mathrm{H}, J=4.8$ and $3.7 \mathrm{~Hz})$.

4.2.11. Compound 3k. Colorless needles, IR $\left(\mathrm{KBr}, \mathrm{cm}^{-1}\right)$ : $3376,3052,2200,1663,1604,1563,1469,1438,1382 ;{ }^{1} \mathrm{H}$ NMR (300 MHz, $\left.\mathrm{CDCl}_{3}, \delta / \mathrm{ppm}\right): 9.28$ (br. s, $1 \mathrm{H}, \mathrm{H}-2$ of 3-pyridyl), $8.50(\mathrm{~d}, 1 \mathrm{H}, J=8.0 \mathrm{~Hz}, \mathrm{H}-4$ of 3 -pyridyl), $8.43(\mathrm{~d}, 1 \mathrm{H}, J=$ $4.6 \mathrm{~Hz}, \mathrm{H}-6$ of 3-pyridyl), 7.54-7.56 (m, $4 \mathrm{H}, o$-proton of 2 $\left.\times \mathrm{C}_{6} \mathrm{H}_{5}\right), 7.28-7.39\left(\mathrm{~m}, 7 \mathrm{H}, m\right.$ - and $p$-protons of $2 \times \mathrm{C}_{6} \mathrm{H}_{5}$ and $\mathrm{H}-5$ of 3-pyridyl); ${ }^{13} \mathrm{C}$ NMR (75 MHz, $\left.\mathrm{CDCl}_{3}, \delta / \mathrm{ppm}\right)$ 148.9, 145.9, 143.1, 133.5, 132.6, 128.6, 128.0, 127.6, 126.7, 123.9; FAB MS $(\mathrm{M}+\mathrm{H})^{+}$: Calcd. 298.3. Found 298.3, Anal. Calcd. for $\mathrm{C}_{20} \mathrm{H}_{15} \mathrm{~N}_{3}$ (297.35): C, 80.78; H, 5.08; N, 14.13. Found: C, 80.56; H, 5.31; N, 13.92.

4.2.12. Compound 31. Colorless needles, ${ }^{1} \mathrm{H}$ NMR $(300 \mathrm{MHz}$, $\left.\mathrm{CDCl}_{3}, \delta / \mathrm{ppm}\right): 7.90(\mathrm{~d}, 2 \mathrm{H}, J=7.8 \mathrm{~Hz}), 7.42-7.48(\mathrm{~m}, 6 \mathrm{H})$, $7.36(\mathrm{t}, 1 \mathrm{H}, J=7.5), 7.16(\mathrm{~d}, 4 \mathrm{H}, J=7.8 \mathrm{~Hz}), 2.36(\mathrm{~s}, 6 \mathrm{H}, 2 \times$ $\left.-\mathrm{CH}_{3}\right)$.

4.2.13. Compound $3 \mathbf{m}$. Colorless needles, ${ }^{1} \mathrm{H} \quad \mathrm{NMR}$ $\left(300 \mathrm{MHz}, \mathrm{CDCl}_{3}, \delta / \mathrm{ppm}\right): 7.83(2 \mathrm{H}, \mathrm{d}, J=8.1 \mathrm{~Hz}), 7.43(\mathrm{~d}$, $4 \mathrm{H}, J=7.8 \mathrm{~Hz}), 7.40(2 \mathrm{H}, \mathrm{d}, J=8.1 \mathrm{~Hz}), 7.14(\mathrm{~d}, 4 \mathrm{H}, J=7.6 \mathrm{~Hz})$, $2.36\left(\mathrm{~s}, 6 \mathrm{H}, 2 \times-\mathrm{CH}_{3}\right)$.
4.2.14. Compound 3n. Colorless needles, ${ }^{1} \mathrm{H} \quad \mathrm{NMR}$ $\left(300 \mathrm{MHz}, \mathrm{CDCl}_{3}, \delta / \mathrm{ppm}\right): 7.83(\mathrm{~d}, 2 \mathrm{H}, J=8.4 \mathrm{~Hz}), 7.43$ (br. d, $4 \mathrm{H}, J=7.2 \mathrm{~Hz}$ ), 7.13 (br. d, $4 \mathrm{H}, J=7.6 \mathrm{~Hz}), 6.96$ (d, $2 \mathrm{H}$, $J=8.5 \mathrm{~Hz}), 3.85\left(\mathrm{~s}, 3 \mathrm{H},-\mathrm{OCH}_{3}\right), 2.36\left(\mathrm{~s}, 6 \mathrm{H}, 2 \times-\mathrm{CH}_{3}\right)$.

\section{Acknowledgments}

Financial assistance from the UGC-CAS and DST-PURSE programs, Department of Chemistry, is gratefully acknowledged. The authors also acknowledge the DST-FIST program of the Department of Chemistry, Jadavpur University, for providing the NMR spectral data. S. Samanta is thankful to the CSIR and S. Sarkar to the UGC, New Delhi, for their research fellowships.

\section{References}

[1] L. Weber, "The application of multi-component reactions in drug discovery," Current Medicinal Chemistry, vol. 9, no. 23, pp. 2085-2093, 2002.

[2] C. Hulme and V. Gore, "Multi-component reactions: emerging chemistry in drug discovery 'from Xylocain to Crixivan," Current Medicinal Chemistry, vol. 10, no. 1, pp. 51-80, 2003.

[3] P. A. Tempest, "Recent advances in heterocycle generation using the efficient Ugi multiple-component condensation reaction," Current Opinion in Drug Discovery and Development, vol. 8, no. 6, pp. 776-788, 2005. 
[4] A. Dömling, "Recent developments in isocyanide based multicomponent reactions in applied chemistry," Chemical Reviews, vol. 106, no. 1, pp. 17-89, 2006.

[5] D. M. D’Souza and T. J. J. Müller, "Multi-component syntheses of heterocycles by transition-metal catalysis," Chemical Society Reviews, vol. 36, no. 7, pp. 1095-1108, 2007.

[6] M. D. S. Duque, A. Christophe, N. Isambert, T. Constantieux, and J. Rodriguez, " $\beta$-diketo building blocks for MCRs-based syntheses of heterocycles," Topics in Heterocyclic Chemistry, vol. 23, pp. 227-277, 2010.

[7] N. J. Liverton, J. W. Butcher, C. F. Claiborne et al., "Design and synthesis of potent, selective, and orally bioavailable tetrasubstituted imidazole inhibitors of p38 mitogen-activated protein kinase," Journal of Medicinal Chemistry, vol. 42, no. 12, pp. 21802190, 1999.

[8] A. K. Takle, M. J. B. Brown, S. Davies et al., "The identification of potent and selective imidazole-based inhibitors of B-Raf kinase," Bioorganic and Medicinal Chemistry Letters, vol. 16, no. 2, pp. 378-381, 2006.

[9] S. E. de Laszlo, C. Hacker, B. Li et al., "Potent, orally absorbed glucagon receptor antagonists," Bioorganic and Medicinal Chemistry Letters, vol. 9, no. 5, pp. 641-646, 1999.

[10] T. Maier, R. Schmierer, K. Bauer, H. Bieringer, H. Buerstell, and B. Sachse, "1-substituted imidazole-5-carboxylic acid derivatives, their preparation and their use as biocides," US Patent 4820335, 1989.

[11] R. Liebl, R. Handte, H. Mildenberger, K. Bauer, and H. Bieringer, "Preparation of 1-phenyl-2-imidazolidinones and -2imidazolidinethiones as herbicides," German Offen. DE 3, 604, 042, 1987, Chemical Abstracts, vol. 108, 6018g, 1988.

[12] L. Wang, K. W. Woods, Q. Li et al., "Potent, orally active heterocycle-based combretastatin A-4 analogues: synthesis, structure-activity relationship, pharmacokinetics, and in vivo antitumor activity evaluation," Journal of Medicinal Chemistry, vol. 45, no. 8, pp. 1697-1711, 2002.

[13] T. F. Gallagher, S. M. Fier-Thompson, R. S. Garigipati et al., "2,4,5-triarylimidazole inhibitors of IL-1 biosynthesis," Bioorganic and Medicinal Chemistry Letters, vol. 5, no. 11, pp. 11711176, 1995.

[14] J. G. Lombardino and E. H. Wiseman, "Preparation and antiinflammatory activity of some nonacidic trisubstituted imidazoles," Journal of Medicinal Chemistry, vol. 17, no. 11, pp. 1182-1188, 1974.

[15] R. A. Turner, C. F. Huebner, and C. R. Scholz, "Studies on imidazole compounds. I. 4-Methylimidazole and related compounds," Journal of the American Chemical Society, vol. 71, no. 8, pp. 2801-2803, 1949.

[16] A. R. Khosropour, "Ultrasound-promoted greener synthesis of 2,4,5-trisubstituted imidazoles catalyzed by $\mathrm{Zr}(\mathrm{acac})_{4}$ under ambient conditions," Ultrasonics Sonochemistry, vol. 15, no. 5, pp. 659-664, 2008.

[17] J. N. Sangshetti, N. D. Kokare, S. A. Kotharkara, and D. B. Shinde, "Ceric ammonium nitrate catalysed three component one-pot efficient synthesis of 2,4,5-triaryl-1H-imidazoles," Journal of Chemical Sciences, vol. 120, no. 5, pp. 463-467, 2008.

[18] B. Karami, K. Eskandari, and A. Ghasemi, "Facile and rapid synthesis of some novel polysubstituted imidazoles by employing magnetic $\mathrm{Fe}_{3} \mathrm{O}_{4}$ nanoparticles as a high efficient catalyst," Turkish Journal of Chemistry, vol. 36, pp. 601-614, 2012.
[19] M. G. Shen, C. Cai, and W. B. Yi, "Ytterbium perfluorooctanesulfonate as an efficient and recoverable catalyst for the synthesis of trisubstituted imidazoles," Journal of Fluorine Chemistry, vol. 129, no. 6, pp. 541-544, 2008.

[20] L. M. Wang, Y. H. Wang, H. Tian, Y. F. Yao, J. H. Shao, and B. Liu, "Ytterbium triflate as an efficient catalyst for one-pot synthesis of substituted imidazoles through three-component condensation of benzil, aldehydes and ammonium acetate," Journal of Fluorine Chemistry, vol. 127, no. 12, pp. 1570-1573, 2006.

[21] S. D. Jadhav, N. D. Kokare, and S. D. Jadhav, "Phosphomolybdic acid catalyzed facile one-pot synthesis of 2,4,5-triaryl- $1 \mathrm{H}$ imidazoles from benzil and aromatic aldehydes," Journal of Heterocyclic Chemistry, vol. 45, no. 5, pp. 1461-1464, 2008.

[22] L. Wang and C. Cai, "Polymer-supported zinc chloride: a highly active and reusable heterogeneous catalyst for one-pot synthesis of 2,4,5-trisubstituted imidazoles," Monatshefte fur Chemie, vol. 140, no. 5, pp. 541-546, 2009.

[23] G. V. M. Sharma, Y. Jyothi, and P. S. Lakshmi, "Efficient roomtemperature synthesis of tri- and tetrasubstituted imidazoles catalyzed by $\mathrm{ZrCl}_{4}$," Synthetic Communications, vol. 36, no. 20, pp. 2991-3000, 2006.

[24] K. Vikrant and M. Ritu, "Synthesis of substituted imidazoles via a multi-component condensation catalyzed by $p$-toluene sulfonic acid, PTSA," Research Journal of Chemical Sciences, vol. 2, no. 4, pp. 18-23, 2012.

[25] G. Brahmachari and S. Das, "A Simple and straightforward method for one-pot synthesis of 2,4,5-triarylimidazoles using titanium dioxide as an ecofriendly and recyclable catalyst under solvent free conditions," Indian Journal of Chemistry, vol. 52, no. 3, pp. 387-393, 2013.

[26] S. Mashkouri and M. R. Naimi-Jamal, "Mechanochemical solvent-free and catalyst-free one-pot synthesis of pyrano[2,3d]pyrimidine-2,4(1H,3H)-diones with quantitative yields," Molecules, vol. 14, no. 1, pp. 474-479, 2009.

[27] P. Galletti, M. Pori, and D. Giacomini, "Catalyst-free Strecker reaction in water: a simple and efficient protocol using acetone cyanohydrin as cyanide source," European Journal of Organic Chemistry, no. 20-21, pp. 3896-3903, 2011.

[28] S. V. Nalage, M. B. Kalyankar, V. S. Patil, S. V. Bhosale, S. U. Deshmukh, and R. P. Pawar, "An efficient noncatalytic protocol for the synthesis of trisubstituted imidazole in polyethylene glycol using microwaves," Open Catalysis Journal, vol. 3, no. 1, pp. 58-61, 2010.

[29] K. F. Shelke, S. B. Sapkal, S. S. Sonar, B. R. Madje, B. B. Shingate, and M. S. Shingare, "An efficient synthesis of 2,4,5-triaryl- $1 H$ imidazole derivatives catalyzed by boric acid in aqueous media under ultrasound-irradiation," Bulletin of the Korean Chemical Society, vol. 30, no. 5, pp. 1057-1060, 2009.

[30] V. S. V. Satyanarayana and A. Sivakumar, "An efficient and novel one-pot synthesis of 2,4,5-triaryl- $1 \mathrm{H}$-imidazoles catalyzed by $\mathrm{UO}_{2}\left(\mathrm{NO}_{3}\right)_{2} \cdot 6 \mathrm{H}_{2} \mathrm{O}$ under heterogeneous conditions," Chemical Papers, vol. 65, no. 4, pp. 519-526, 2011.

[31] A. Parveen, M. R. S. Ahmed, K. A. Shaikh, S. P. Deshmukh, and R. P. Pawar, "Efficient synthesis of 2,4,5-triaryl substituted imidazoles under solvent free conditions at room temperature," Arkivoc, vol. 2007, no. 16, pp. 12-18, 2007. 
[32] J. F. Zhou, G. X. Gong, X. J. Sun, and Y. L. Zhu, "Facile method for one-step synthesis of 2,4,5-triarylimidazoles under catalystfree, solvent-free, and microwave-irradiation conditions," Synthetic Communications, vol. 40, no. 8, pp. 1134-1141, 2010.

[33] S. Samai, G. C. Nandi, P. Singh, and M. S. Singh, "L-proline: an efficient catalyst for the one-pot synthesis of 2,4,5-trisubstituted and 1,2,4,5-tetrasubstituted imidazoles," Tetrahedron, vol. 65, no. 49, pp. 10155-10161, 2009. 

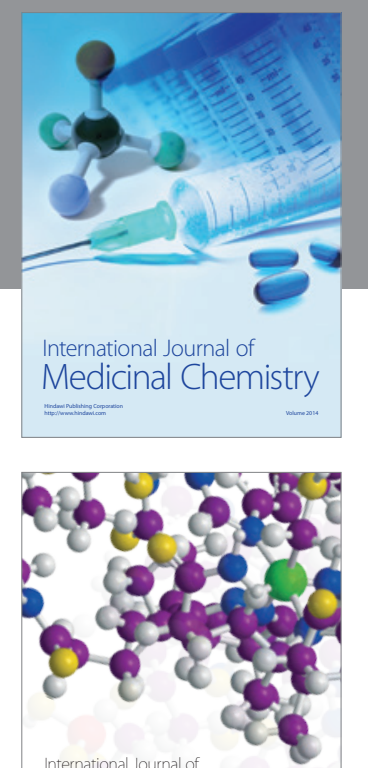

\section{Carbohydrate} Chemistry

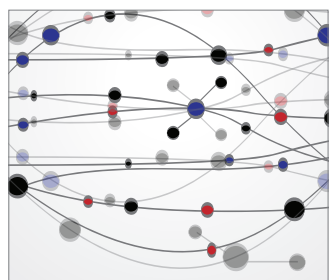

The Scientific World Journal
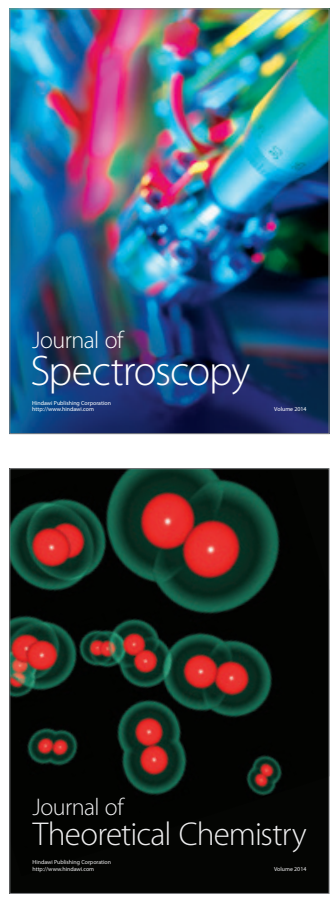
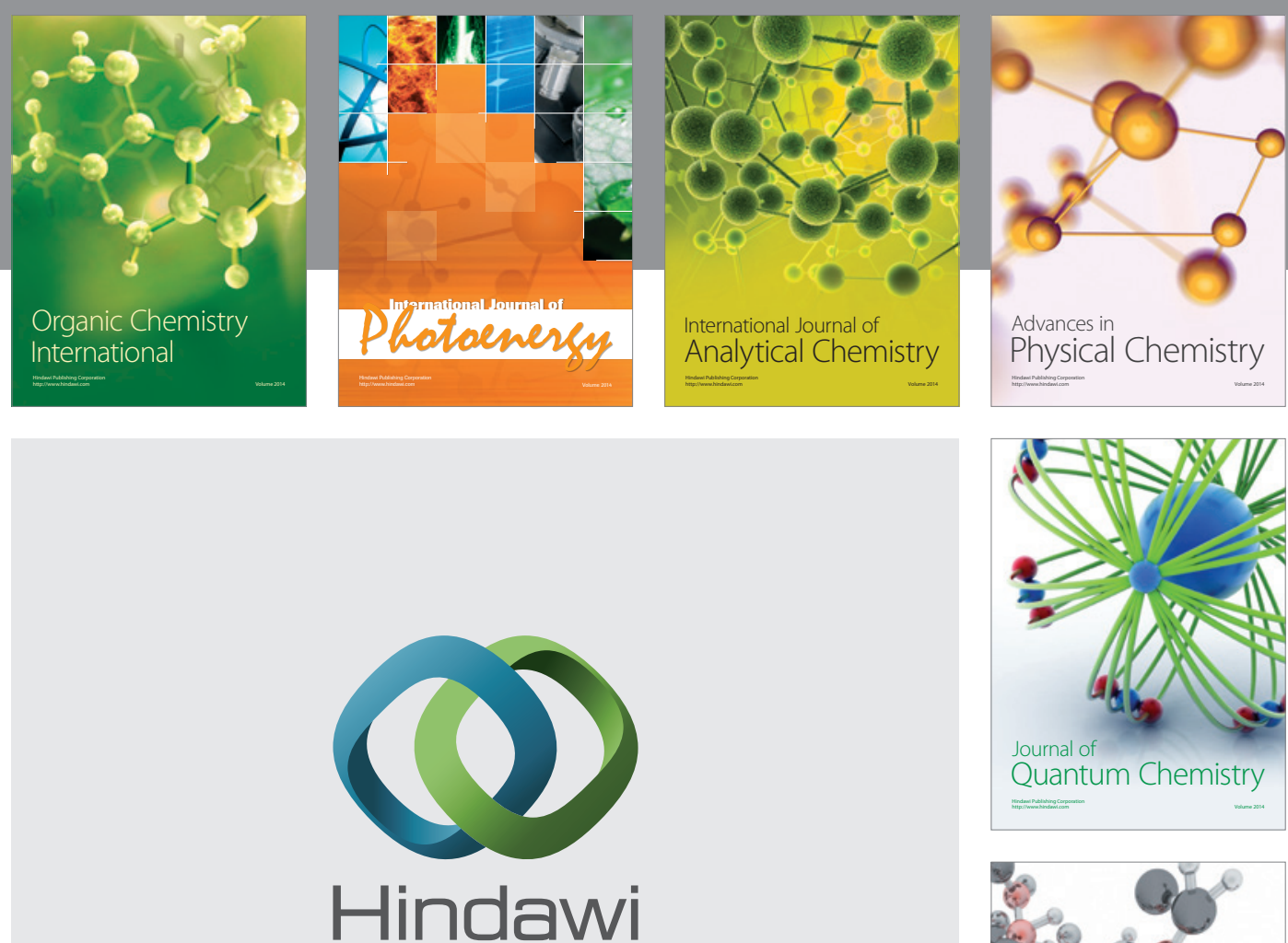

Submit your manuscripts at

http://www.hindawi.com

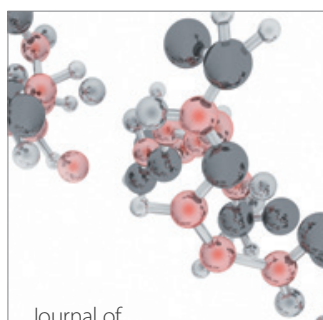

Analytical Methods

in Chemistry

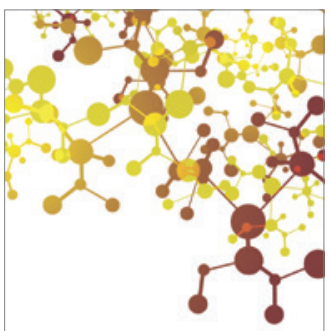

Journal of

Applied Chemistry

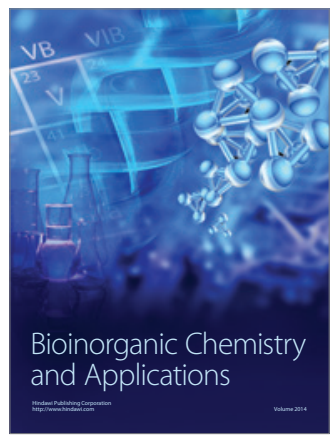

Inorganic Chemistry
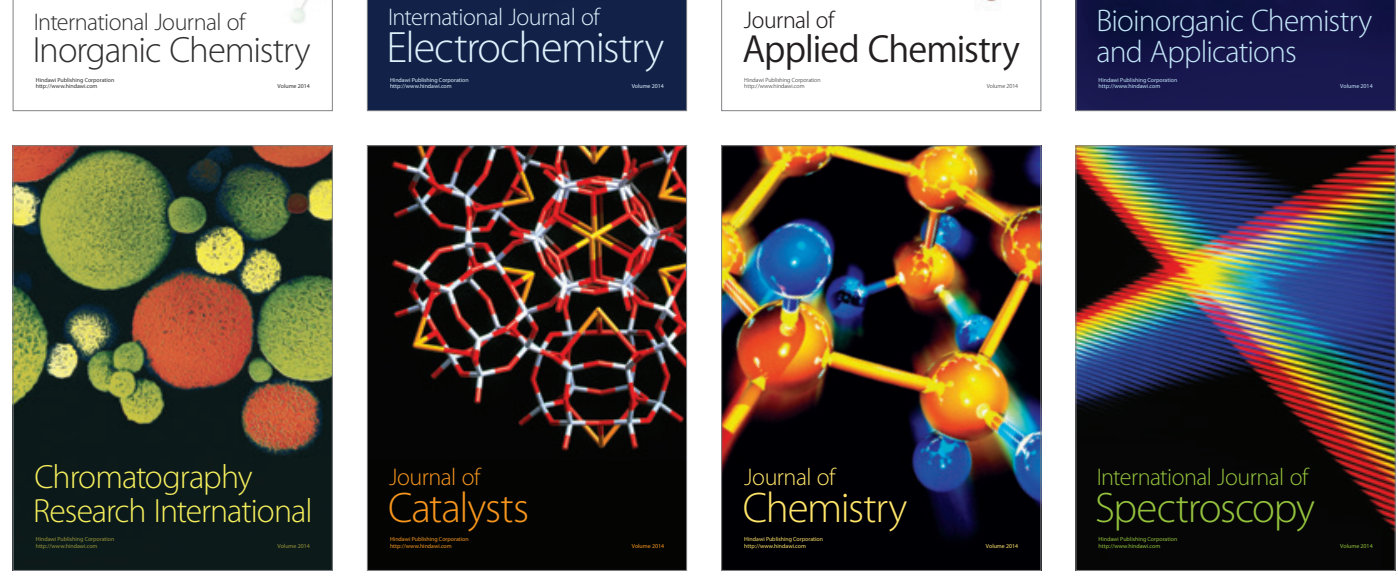